\title{
A Case Report on Anal Cancer
}

Shivani Modi

American International Medical University, Saint Lucia. Email: shvnmd@gmail.com

DOI: http://doi.org/10.38177/AJBSR.2021.3208

Copyright: (02021 Shivani Modi. This is an open access article distributed under the terms of the Creative Commons Attribution License, which permits unrestricted use, distribution, and reproduction in any medium, provided the original author and source are credited.

\section{ABSTRACT}

I report a case of rare type of anal cancer in a relatively young individual. A 35-year-old male presented to the OPD Clinic from Nigeria with pain in anal region. Patient was referred for CT scan which showed anal mass. Biopsy showed highly aggressive adenocarcinoma with no metastasis. Patient was started on radiation therapy and multi-drug regimen chemotherapy. After receiving 2 appointments of radiation therapy, patien contracted community acquired pneumonia. Patient's condition deteriorated and was admitted to ICU for 15 days aggressively treated with antibiotics. Patient was scheduled to undergo abdominoperineal resection. Financially drained and post-surgery complications, patient decided on not receiving any further treatment and returning back home.

Keywords: Abdominoperineal resection, Anal cancer, Adenocarcinoma, Pneumonia, Radiotherapy.

\section{Introduction}

Squamous cell carcinomas account for about $75 \%$ of anal canal tumors, while adenocarcinomas account for over $20 \%{ }^{[1,2]}$. Adenocarcinoma of the anal canal is a type of adenocarcinoma that develops in the anal canal's epithelium, which includes the mucosal surface, anal glands, and the lining of fistulous tracts. Smoking, HIV infection, homosexuality, and anal Crohn's are all risk factors for the development of anal adenocarcinoma. Colorectal cancer originating in the anal mucosa above the dentate line or extra mucosal (perianal) adenocarcinoma are two possibilities ${ }^{[5,6]}$. Extra mucosal adenocarcinoma is a rare type of adenocarcinoma that is defined by extra mucosal or intramural development with no luminal component. Because the tumor invades the submucosa so thoroughly, it can be misinterpreted for metastatic gastrointestinal cancer because the underlying mucosa is unaffected. These tumors can develop from anal glands, which are mucus-producing branched tubular structures with ducts that open at the dentate line, or they can develop from a pre-existing anal fistula ${ }^{[2,4]}$.

\section{Case Presentation}

A 35-year-old male patient presented to the OPD Clinic from Nigeria with C/O pain in the anal region, no bleeding PR. He has H/O of obstructive neuropathy with serum creatinine 16. He underwent bilateral nephrostomy and serum creatinine decreased to 6 . Now he is admitted for further management. His past medical history includes bilateral nephrostomy, hypertension, and medications, and ESRD, and Dialysis. Upon examination: he was afebrile, vitals: stable

CVS: S1S2 normal

RS: AEBE, clear

P/A: soft, non-tender

CNS: conscious, oriented

P/R: ulcer proliferative growth in anal cancer

Circumferential, unable to insert a finger 
He was admitted to the hospital. Relevant investigations were done which include CBC, electrolyte, Chest X-ray, urine routine, and urine culture. Preventative measures were taken like avoiding colostomy wound contamination and the patient was left on inotropic support, IVF, IV antibiotics. He underwent an MRI of the abdomen and pelvis w/o contrast. Findings of MRI include diffuse nodular soft tissue thickening of the rectum for a length of approximately $7 \mathrm{~cm}$. The thickening starts approximately $4 \mathrm{~cm}$ from the anal verge. There is an extension of the lesion into the prostate and seminal vesicles. The fat plane between the rectum and prostate is lost. The growth is seen near the right lateral wall. The right lateral wall shows focal thinning and a bulge. No obvious collection is seen adjacent to this. The liver is enlarged in size $(18 \mathrm{~cm}$ in MCL) and shows diffuse hypotenuse $\mathrm{T} 2$ signal. Hyperintense signal is seen in the out-phase images suggestive of iron deposition. Spleen (14.8) is enlarged in size. Pancreas, gallbladder, intra and extrahepatic bile ducts are unremarkable. Both adrenals and kidneys are of normal size and shape. Both the lower ureters are dilated. The urinary bladder is partially distended and shows frost bladder wall thickening. No size significant lymphadenopathy is seen. Visualized small bowel loops appear unremarkable. Bones appear unremarkable. 2D/3D Echo with Doppler was performed on the patient. The findings were as follows.

\section{LVEF: $25 \%$}

Severe LV systolic dysfunction

Fair RV systolic function

Global LV hypokinesia

No clot/vegetation

Thin rim of pericardial effusion. Pleural effusion noted.

Tip of port seen in RA near SVC.

Plan of treatment and other options were discussed with the patient. He underwent EUA Rectum + Biopsy + De functioning colostomy under general anaesthesia. The procedure went uneventful. The patient clinically stable hence is being discharged with a stoma bag.

\section{Biopsy report shows:}

Gross description: Received multiple grey-brown soft fragments aggregating to $2 \mathrm{~cm}$.

Microscopic description: Adenocarcinoma of anal cancer

This tumor infiltrates into the muscular propria. Perineural invasion is seen.

The stage of the tumor is T4N?M0.

He was undergoing radiation to shrink the tumor. After successful completion of radiation, the patient needs to undergo abdominoperineal resection. Abdominoperineal resection is the procedure for removal of the lower part of the rectum, prostate, seminal vesicle, and anal canal. After this procedure, he will permanently have a colostomy bag for stools and uro-bag for urine. Abdominoperineal Resection is a major surgical procedure that carries the risk 
of complications. General complications include Infection in a wound, bleeding from wound or operation site, chest infection, DVT. He might also require staying in the hospital for 7-12 days after the surgery.

He was considering the treatment option in the meanwhile he acquired iatrogenic pneumonia. He was admitted to the ICU for treatment. His WBC count was low, so he was monitored for the same. He was given IV antibiotics. He recovered from pneumonia and his WBC count increased within 10-12 days. He was too fed up with the situation and decided to not undergo surgical removal of the tumor. The patient completed the radiation therapy and went back to Nigeria with the colostomy bag.

\section{Discussion}

Anal Cancer is an uncommon malignancy that starts at the anus. Due to the large area, it is until late in the disease process when patient experiences symptoms. Anal cancer is prevalent in older men but rarely also found in men older than 35 years. Symptoms of anal cancer include bleeding from the anus, itchy feeling, mass in anus along pain. Later in the disease course, obstructive nephropathy can be seen. Risk factors include smoking, HPV, anal sex, multiple sexual partners ${ }^{[2,4,7]}$. It is very important to make a diagnosis once anal cancer is suspected. Compared to adenocarcinoma, Squamous cell carcinoma of the anus has a better prognosis overall ${ }^{[5]}$.

An abdominoperineal resection (APR) was once a frequent treatment for anal cancer, but doctors have discovered that radiation therapy and chemotherapy may nearly always replace it. APR is now only used if other treatments have failed or if cancer has returned following treatment. An APR is a significant operation. To remove the anus, the rectum, and the sigmoid colon, the surgeon makes one incision (cut) in the abdomen (belly) and another around the anus. The surgeon may also do a lymph node dissection to remove some of the adjacent lymph nodes in the groin area, though this can be done later. Because the anus (and the anal sphincter) has been removed, a new hole must be created for stool to exit the body ${ }^{[1,3]}$.

The end of the colon is connected to a small hole (called a stoma) in the abdomen to do this. A bag for collecting faeces is attached to the body and placed over the hole. APR has a higher rate of adverse effects, many of which are long-term. Following an APR, you may develop scar tissue (adhesions) in your abdomen, which can cause organs or tissues to cling together. This might result in pain or difficulty moving food through the bowels, which can lead to digestive issues. An APR also necessitates the use of a permanent colostomy. It may take some time to adjust to this, and it may necessitate certain lifestyle changes. The ureters or urethra (tubes that collect urine) might be damaged by an APR, making it difficult to urinate (pee). This may necessitate additional surgery ${ }^{[3,7]}$.

\section{Conclusion}

From the above case report, it is evident that diagnosis made earlier in the disease course is highly treatable. In this patient, it is evident that lack of advanced medical instruments and belonging to an underdeveloped country can pose significant challenges in the diagnosis along with treatment course. One of the reasons the patient opt-out of abdominoperineal resection was the financial crisis. Also, the fact cannot be neglected that delay in seeking treatment was one of the major setbacks for this patient. Radiotherapy has an extra effect and is still a popular alternative for gaining local control and alleviating symptoms. To establish the therapeutic method to be used, a 
thorough histological investigation and immunohistochemistry are required. Considering all this, I strongly believe that an awareness campaign for anal cancer should be implicated more in an underdeveloped country. Further need to make hospital fully equipped should be considered by the Government.

\section{Declarations}

\section{Source of Funding}

This research did not receive any grant from funding agencies in the public, commercial, or not-for-profit sectors.

\section{Competing Interests Statement}

The author declares no competing financial, professional and personal interests.

\section{Ethical Approval}

Ethical approval for this research was given based on guidelines from American International Medical University.

\section{Patient consent for publication}

Not required.

\section{Availability of data and material}

Author is willing to share data and material according to the relevant needs.

\footnotetext{
References

[1] https://www.mayoclinic.org/diseases-conditions/anal-cancer/symptoms-causes/syc-20354140.

[2] https://www.webmd.com/cancer/what-is-anal-cancer\#1.

[3] https://www.cancer.org/cancer/anal-cancer.html.

[4] https://www.ncbi.nlm.nih.gov/pmc/articles/PMC3369602/\#: :text=Adenocarcinomas\%20make\%20up\%202. 9\%25\%E2\%80\%9310\%25\%20of\%20anal\%20cancers.\&text=Anal\%20adenocarcinoma\%20(AA)\%20is\%20an, than\%20anal\%20squamous\%20cell\%20cancer.

[5] Rodney E. Wegner, Richard J. White, Shaakir Hasan, Moses Raj, Dulabh Monga, Gene Finley, Alexander V. Kirichenko, James Mc Cormick, “Anal adenocarcinoma: Treatment outcomes and trends in a rare disease entity”, s. Cancer Medicine, DOI: 10.1002/cam4.2076.

[6] Tsay CJ, Pointer T, Chandler JB, et al. Anal adenocarcinoma: case report, literature review and comparative survival analysis. BMJ Open Gastro 2021;8:e000661. doi:10.1136/bmjgast-2021-000661

[7] https://www.healthline.com/health/anal-cancer.
} 